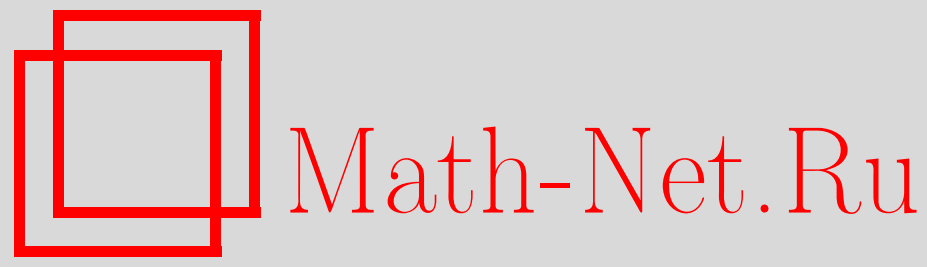

Ал. И. Буфетов, Центральная предельная теорема для экстремальных характеров бесконечной симметрической группы, Функи. анализ и его прил., 2012, том 46, выпуск 2, 3-16

DOI: https://doi.org/10.4213/faa3075

Использование Общероссийского математического портала MathNet.Ru подразумевает, что вы прочитали и согласны с пользовательским соглашением

http://www . mathnet.ru/rus/agreement

Параметры загрузки:

IP : 54.224 .60 .19

26 апреля 2023 г., 17:42:55

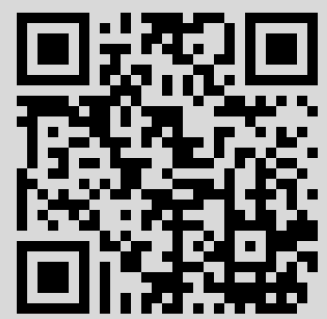


Функционалъный анализ и его приложения

2012, т. 46, вып. 2, с. 3-16

УДК 519.21

\title{
Центральная предельная теорема для экстремальных характеров бесконечной симметрической группы*
}

\author{
(C) 2012. АЛЕКСЕЙ И. БУФЕТОВ
}

В работе изучается предельное поведение длин первых строк и столбцов случайных диаграмм Юнга, соответствующих экстремальным характерам бесконечной симметрической группы. Мы рассматриваем линейно растущие строки и столбцы и доказываем для их длин, при некоторых ограничениях, центральную предельную теорему. Мы также устанавливаем более точное утверждение, связывающее рост строк и столбцов с простой моделью с независимыми испытаниями.

\section{§1. Введение}

Пусть $\mathbb{Y}_{n}$ - множество диаграмм Юнга из $n$ клеток. Определим градуированный граф Юнга $\mathbb{Y}$, множеством вершин которого служит $\bigcup_{n=1}^{\infty} \mathbb{Y}_{n}$, а ребро между диаграммами $\lambda$ и $\mu$ проведено в том и только в том случае, когда $\lambda \in \mathbb{Y}_{n}$, $\mu \in \mathbb{Y}_{n+1}$ и $\mu$ получается из $\lambda$ добавлением одной клетки (в этом случае будем писать $\lambda \uparrow \mu)$. Пусть $\operatorname{dim} \lambda$ - число различных кратчайших путей в $\mathbb{Y}$ от одноклеточной диаграммы Юнга до диаграммы $\lambda$.

Когерентной системой мер на $\mathbb{Y}$ называется последовательность $\left\{M_{n}\right\}$, где $M_{n}$ - вероятностная мера на $\mathbb{Y}_{n}$, для которой выполнено соотношение

$$
M_{n}(\nu)=\sum_{\lambda: \nu \uparrow \lambda} \frac{\operatorname{dim} \nu}{\operatorname{dim} \lambda} M_{n+1}(\lambda) \quad \text { для любого } \nu \in \mathbb{Y}_{n} .
$$

Хорошо известно, что характеры бесконечной симметрической группы взаимно однозначно соответствуют когерентным системам мер на $\mathbb{Y}$. По теореме Тома (см. [1]) экстремальные характеры описываются наборами параметров $\mathscr{P}=\left(\left\{\alpha_{i}\right\},\left\{\beta_{j}\right\}, \gamma\right)$, где $\alpha_{i}, \beta_{j}, \gamma-$ вещественные числа, удовлетворяющие соотношениям

$$
\alpha_{1} \geqslant \alpha_{2} \geqslant \cdots \geqslant 0, \quad \beta_{1} \geqslant \beta_{2} \geqslant \cdots \geqslant 0, \quad \gamma \geqslant 0, \quad \sum_{i=1}^{\infty}\left(\alpha_{i}+\beta_{i}\right)+\gamma=1 .
$$

Пусть $\left\{M_{n}^{\mathscr{P}}\right\}$ - когерентная система мер, соответствующая фиксированному набору параметров $\mathscr{P}$. Обозначим символом $\lambda_{i}^{\mathscr{P}}(n)$ длину $i$-й строки случайной диаграммы Юнга, выбранной по мере $M_{n}^{\mathscr{P}}$, а символом $\lambda_{j}^{\prime \mathscr{P}}(n)-$ длину $j$-го столбца этой диаграммы. Нашей основной задачей является изучение асимптотического поведения этих величин.

*Работа выполнена при финансовой поддержке фонда Саймонса, фонда поддержки молодых ученых «Конкурс Мёбиуса» и гранта РФФИ-НЦНИЛ-10-01-93114. 
Известно (см. [4]-[6]), что для длин строк и столбцов выполнен закон больших чисел:

$$
\frac{\lambda_{i}^{\mathscr{P}}(n)}{n} \underset{\mathrm{prob}}{\longrightarrow} \alpha_{i}, \quad \frac{\lambda_{j}^{\prime \mathscr{P}}(n)}{n} \underset{\mathrm{prob}}{\longrightarrow} \beta_{j} .
$$

Основным результатом настоящей работы является центральная предельная теорема для случая строго монотонных последовательностей $\left\{\alpha_{i}\right\},\left\{\beta_{j}\right\}$. Впервые такого рода результат был получен Ферэ и Мелио [2] для случая $\alpha_{i}=$ $(1-q) q^{i-1}, \beta_{j}=0, \gamma=0 ;$ их статья послужила толчком к настоящей работе, но наш метод иной. Для случая произвольных строго монотонных последовательностей $\alpha_{i}, \beta_{j}$, а также для случая произвольных параметров $\left\{\alpha_{i}\right\}$ при $\beta_{j}=0$, $\gamma=0$ центральная предельная теорема была независимо доказана Мелио в [3]. Настоящая работа была закончена и подана в журнал до появления препринта [3] в архиве.

Будем рассматривать наборы параметров $\mathscr{P}$, для которых

$$
\begin{aligned}
& \alpha_{i}>\alpha_{i+1} \text { для всех } i, \text { таких, что } \alpha_{i} \neq 0, \\
& \beta_{j}>\beta_{j+1} \text { для всех } j, \text { таких, что } \beta_{j} \neq 0 .
\end{aligned}
$$

Заметим, что условие неравенства параметров существенно: например, если $\alpha_{1}=\cdots=\alpha_{k}=1 / k$, то флуктуации не являются гауссовыми (см. [14], [15]).

Теорема 1 (центральная предельная теорема). Пусть Я - произвольныи набор параметров, удовлетворяющий условиям (1.1), и $K, L>0$ таковъ, что $\alpha_{1}>\cdots>\alpha_{K}>0$ u $\beta_{1}>\cdots>\beta_{L}>0$. Тогда

$$
\begin{aligned}
\left(\frac{\lambda_{1}^{\mathscr{P}}(n)-\alpha_{1} n}{\sqrt{n}}, \ldots, \frac{\lambda_{K}^{\mathscr{P}}(n)-\alpha_{K} n}{\sqrt{n}}, \frac{\lambda_{1}^{\prime \mathscr{P}}(n)-\beta_{1} n}{\sqrt{n}}, \ldots, \frac{\lambda_{L}^{\prime \mathscr{P}}(n)-\beta_{L} n}{\sqrt{n}}\right) \\
\underset{\text { law }}{\longrightarrow} Z=\left(Z_{1}, \ldots, Z_{k}, Z_{1}^{\prime}, \ldots, Z_{L}^{\prime}\right),
\end{aligned}
$$

где $Z$ - многомерная гауссова случайная величина с моментами

$$
\begin{gathered}
\mathbf{E} Z_{i}=0, \quad \mathbf{E} Z_{i}^{\prime}=0, \\
\mathbf{E} Z_{i}^{2}=\alpha_{i}-\alpha_{i}^{2}, \quad \mathbf{E} Z_{i}^{\prime 2}=\beta_{i}-\beta_{i}^{2}, \\
\mathbf{E} Z_{i} Z_{j}=-\alpha_{i} \alpha_{j}, \quad \mathbf{E} Z_{i}^{\prime} Z_{j}^{\prime}=-\beta_{i} \beta_{j}, \quad \mathbf{E} Z_{i} Z_{j}^{\prime}=-\alpha_{i} \beta_{j} .
\end{gathered}
$$

Замечание 1. Пусть $\left\{X_{i}\right\},\left\{Y_{j}\right\}, \Theta$ - независимые в совокупности гауссовы случайные величины с нулевым средним и дисперсиями

$$
\mathbf{E} X_{i}^{2}=\alpha_{i}, \quad \mathbf{E} Y_{j}^{2}=\beta_{j}, \quad \mathbf{E} \Theta^{2}=\gamma,
$$

при этом $X_{i}, Y_{j}$ определены для всех ненулевых $\alpha$ - и $\beta$-параметров. Тогда распределение $\left(Z_{1}, \ldots, Z_{K}, Z_{1}^{\prime}, \ldots, Z_{L}^{\prime}\right)$ совпадает с проекцией на первые $K+L$ координат условного распределения на гиперплоскости $X_{1}+\cdots+X_{K}+X_{K+1}+$ $\cdots+Y_{1}+\cdots+Y_{L}+Y_{L+1}+\cdots+\Theta=0$.

Замечание 2. Пусть $\widetilde{M}_{\nu}^{\mathscr{P}}-$ мера на $\mathbb{Y}$, являющаяся пуассонизацией последовательности мер $M_{n}^{\mathscr{P}}$,

$$
\widetilde{M}_{\nu}^{\mathscr{P}}(\lambda):=e^{-\nu} \frac{\nu^{|\lambda|}}{|\lambda| !} M_{|\lambda|}^{\mathscr{P}}(\lambda),
$$


где символом $|\lambda|$ обозначено число клеток в диаграмме $\lambda$, и $\tilde{\lambda}_{i}^{\mathscr{P}}(\nu), \tilde{\lambda}_{j}^{\prime P}(\nu)-$ длины $i$-й строки и $j$-го столбца случайной диаграммы Юнга, взятой по этой мере. В условиях теоремы 1

$$
\begin{aligned}
\left(\frac{\tilde{\lambda}_{1}^{\mathscr{P}}(\nu)-\alpha_{1} \nu}{\sqrt{\nu}}, \ldots, \frac{\tilde{\lambda}_{K}^{\mathscr{P}}(\nu)-\alpha_{K} \nu}{\sqrt{\nu}}, \frac{\tilde{\lambda}_{1}^{\prime \mathscr{P}}(\nu)-\beta_{1} \nu}{\sqrt{\nu}}, \ldots, \frac{\tilde{\lambda}_{L}^{\prime \mathscr{P}}(\nu)-\beta_{L} \nu}{\sqrt{\nu}}\right) \\
\underset{\text { law }}{\stackrel{\nu \rightarrow \infty}{\longrightarrow}}\left(X_{1}, \ldots, X_{K}, Y_{1}, \ldots, Y_{L}\right)
\end{aligned}
$$

Доказательство аналогично доказательству теоремы 1.

Пусть $\mathscr{A}$ - алфавит, состоящий из дискретной части - множеств $L_{e}=$ $\left\{x_{1}, x_{2}, \ldots\right\}$ и $L_{o}=\left\{y_{1}, y_{2}, \ldots\right\}$ - и непрерывной части $G$, которую будем считать отрезком. Введем на $\mathscr{A}$ вероятностную меру $\mu_{1}$, сопоставляя букве $x_{i}$ вероятность $\alpha_{i}$, букве $y_{j}$ - вероятность $\beta_{j}$ и считая, что на $G$ задана мера Лебега с условием $\mu_{1}(G)=\gamma$; зададим на $\mathscr{A}^{n}$ бернуллиевскую меру $\mu_{n}=\mu_{1}^{\otimes n}$. Обозначим символом $N_{x_{i}}(n)$ случайную величину, равную числу букв $x_{i}$ в случайном слове $w \in \mathscr{A}^{n}$, выбранном по мере $\mu_{n}$, а символом $N_{y_{j}}(n)$ - число букв $y_{j}$ в этом слове. Будем считать, что на $\mathscr{A}$ введено некоторое линейное упорядочение $p$. Как было показано в [7], с помощью обобщенного RSK-алгоритма можно построить отображение

$$
\phi_{p}: \mathscr{A}^{n} \rightarrow \mathbb{Y}_{n},
$$

такое, что мера $\mu_{n}$ под действием $\phi_{p}$ переходит в меру $M_{n}^{\mathscr{P}}$. В силу этого можно считать, что величины $\lambda_{i}^{\mathscr{P}}(n), \lambda_{j}^{\prime \mathscr{P}}(n)$ заданы на вероятностном пространстве $\left(\mathscr{A}^{n}, \mu_{n}\right)$.

Теорема 2. Пусть ЯР - произвольный набор параметров, удовлетворяющий условиям (1.1), и $K, L>0$ таковы, что $\alpha_{1}>\cdots>\alpha_{K}>0 u \beta_{1}>\cdots>$ $\beta_{L}>0$. Определим функиии

$$
\begin{array}{rlrl}
\epsilon_{1}(n):=\lambda_{1}^{\mathscr{P}}(n)-N_{x_{1}}(n), & \ldots, & \epsilon_{K}(n):=\lambda_{K}^{\mathscr{P}}(n)-N_{x_{K}}(n), \\
\epsilon_{1}^{\prime}(n):=\lambda_{1}^{\prime \mathscr{P}}(n)-N_{y_{1}}(n), \quad \ldots, & \epsilon_{L}^{\prime}(n):=\lambda_{L}^{\prime \mathscr{P}}(n)-N_{y_{L}}(n) .
\end{array}
$$

Тогда существует константа $C=C(K, L)$ (не зависящая от $n$ ), такая, что

$$
\mathbf{E}\left|\epsilon_{i}(n)\right|<C, \quad \mathbf{E}\left|\epsilon_{j}^{\prime}(n)\right|<C, \quad i=1, \ldots, K, j=1, \ldots, L .
$$

Теорема 1 является простым следствием теоремы 2.

Замечание 3. В определении величин $\lambda_{i}^{\mathscr{P}}(n)$ на пространстве $\left(\mathscr{A}^{n}, \mu_{n}\right)$ имеется неоднозначность, связанная с произвольностью выбора линейного упорядочения алфавита $\mathscr{A}$. Как будет показано в разд. 2.2 , этот произвол не влияет на распределение величин $\lambda_{i}^{\mathscr{P}}(n)-N_{x_{i}}(n)$.

Замечание 4. Пусть $\tilde{\mu}_{\nu}(w)$ - мера на словах произвольной длины из букв алфавита $\mathscr{A}$, определяемая по формуле

$$
\tilde{\mu}_{\nu}(w):=\frac{\nu^{|w|}}{|w| !} \mu_{|w|}(w),
$$

где $|w|$ — число букв в слове $w$. Будем обозначать символом $\widetilde{N}_{x_{i}}(\nu)$ (соответственно $\left.\widetilde{N}_{y_{j}}(\nu)\right)$ число букв $x_{i}$ (соответственно $\left.y_{j}\right)$ в случайном слове $w$, взятом по мере $\tilde{\mu}_{\nu}$. В тех же предположениях утверждение теоремы 2 выполнено для 
разностей $\tilde{\lambda}_{i}^{\mathscr{P}}(\nu)-\widetilde{N}_{x_{i}}(\nu), \tilde{\lambda}_{j}^{\prime \mathscr{P}}(\nu)-\tilde{N}_{y_{j}}(\nu)$. Доказательство аналогично доказательству теоремы 2 .

Замечание 5. Теорема 2 может быть переформулирована в терминах, не использующих RSK-алгоритм. Для этого величины $\lambda_{i}^{\mathscr{P}}(n), \lambda_{j}^{\prime \mathscr{P}}(n)$ следует определять на вероятностном пространстве, состоящем из $\mathscr{A}_{p}$-таблиц (см. определение в разд. 2.1).

Автор глубоко благодарен Г. И. Ольшанскому и А. М. Бородину за постановку задачи и многочисленные полезные обсуждения. Автор благодарен Л. А. Петрову за замечания, способствовавшие улучшению текста.

\section{§2. Основные леммы}

2.1. В этом разделе мы изложим необходимый нам в дальнейшем материал из [7].

Пусть на алфавите $\mathscr{A}$ задано линейное упорядочение $p$. Будем писать $x \nearrow y$, если $x<y$ или $x=y \in L_{e}$, и $x \searrow y$, если $x>y$ или $x=y \in L_{o} \cup G$. Назовем слово $w=x_{1} \ldots x_{n}$ возрастающим, если $x_{1} \nearrow \ldots \nearrow x_{n}$, и убъвающим, если $x_{1} \searrow \cdots \searrow x_{n}$. Определим $\mathscr{A}_{p}$-таблицу формы $\lambda$ как диаграмму Юнга $\lambda$, заполненную буквами из $\mathscr{A}$, в которой вдоль строк стоят возрастающие слова, а вдоль столбцов, читаемых снизу вверх, - убывающие (см. пример ниже). Обобщенный RSK-алгоритм сопоставляет слову $w \in \mathscr{A}^{n}$ пару $(R(w), S(w))$, где $R(w)$ есть $\mathscr{A}_{p}$-таблица, а $S(w)$ - стандартная ${ }^{1)}$ таблица Юнга, причем $R(w)$ и $S(w)$ имеют одну и ту же форму $\lambda$. Отображение

$$
\phi_{p}: \mathscr{A}^{n} \rightarrow \mathbb{Y}_{n}
$$

определяется как сопоставление слову $w$ этой формы $\lambda$. Опишем действие обобщенного RSK-алгоритма.

Определим сначала алгоритм строчной вставки; по $\mathscr{A}_{p}$-таблице $T$ и букве $x \in \mathscr{A}$ он строит новую $\mathscr{A}_{p}$-таблицу, которая обозначается через $x \rightarrow T$. Эта таблица содержит на одну клетку больше, чем $T$, а множество ее элементов это те же элементы, что и в $T$, а также элемент $x$. Предположим, что $x \in L_{e}$. Тогда расстановка этих элементов определяется следующим образом: если $x$ больше или равен каждому элементу из первой строки, то просто ставим его в новую клетку в конце первой строки. В противном случае находим самый маленький элемент первой строки, строго превосходящий $x$. Выбиваем этот элемент из клетки и ставим $x$ на его место. Если же $x \in L_{o}$, то правило аналогично, только $x$ может выбивать не только строго большие элементы, но и равные себе. С выбитым элементом повторяем те же действия относительно второй строки. Продолжаем этот процесс, пока очередной выбитый элемент не оказывается в конце очередной строки или не выбивается из последней строки - в этом случае образуем новую строку из одного элемента.

Для $w=x_{1} \cdots x_{n}$ определим $R(w)$ формулой

$$
R(w):=\left[\left(x_{n} \rightarrow\left(x_{n-1} \rightarrow\left(x_{n-2} \cdots \rightarrow\left(x_{2} \rightarrow\left(x_{1} \rightarrow \varnothing\right)\right) \ldots\right)\right] .\right.\right.
$$

1) Стандартной таблицей Юнга называется диаграмма $\lambda$, заполненная числами от 1 до $|\lambda|$, каждое из которых встречается ровно по одному разу, и вдоль всех строк и столбцов которой стоят возрастающие последовательности. 
На каждом шаге алгоритма к $R(w)$ присоединяется одна новая клетка. Таблица Юнга $S(w)$ определяется нумерацией клеток в порядке их присоединения к $R(w)$.

Пример. Пусть $x_{1}<x_{2}<y_{1}<y_{2}$ и $L_{e}=\left\{x_{1}, x_{2}\right\}, L_{o}=\left\{y_{1}, y_{2}\right\}$. Тогда слово $w=x_{1} y_{1} y_{1} y_{2} x_{2} x_{1} y_{1}$ под действием обобщенного RSK-алгоритма перейдет в пару таблиц

\begin{tabular}{|l|l|l|}
\hline$x_{1}$ & $x_{1}$ & $y_{1}$ \\
\hline$x_{2}$ & $y_{2}$ & \\
\cline { 1 - 1 }$y_{1}$ & \multicolumn{2}{|}{} \\
\cline { 1 - 1 }$y_{1}$ & \multicolumn{2}{|}{} \\
\cline { 1 - 1 }
\end{tabular}

\begin{tabular}{|l|l|l|}
\hline 1 & 2 & 4 \\
\cline { 1 - 2 } 3 & 7 & \multicolumn{1}{|l}{} \\
\cline { 1 - 1 } 5 & \multicolumn{2}{|}{} \\
\cline { 1 - 1 } 6 & \multicolumn{2}{|l}{} \\
\cline { 1 - 1 } &
\end{tabular}

Обозначим максимальное натуральное число, которое можно получить как сумму длин $k$ непересекающихся возрастающих (соответственно убывающих) подпоследовательностей слова $w$, символом $r_{k}(w)$ (соответственно $\left.c_{k}(w)\right)$.

Утверждение 1. (а) Обобщенный $R S K$-алгоритм задает биекиию между $\mathscr{A}^{n}$ и парами $(R, S)$, где $R$ есть $\mathscr{A}_{p}$-таблища, $S$ - таблица Юнга $u R, S$ имеют общую форму, состоящую из $n$ клеток.

(b) Выполнень равенства

$$
r_{k}(w)=\sum_{i=1}^{k} \lambda_{i}\left(\phi_{p}(w)\right), \quad c_{k}(w)=\sum_{j=1}^{k} \lambda_{j}^{\prime}\left(\phi_{p}(w)\right) .
$$

Доказательство. Это утверждение является обобщением теоремы Шенстеда (см. [12]). Как указано в [7, предложение 1], доказательство аналогично доказательству теоремы Шенстеда (см., например, [11]).

Пусть $\Lambda$ - алгебра симметрических функций от бесконечного числа переменных (см. [8, гл. 1.2]). Обозначим символом $h_{n}$ полные однородные симметрические функции, а символом $s_{\lambda}-$ функции Шура. Определим производящую функцию элементов $h_{n}$ формулой

$$
H(z)=1+\sum_{n=1}^{\infty} h_{n} z^{n}
$$

и пусть $\pi^{\mathscr{P}}: \Lambda \rightarrow \mathbb{C}$ - гомоморфизм, задаваемый на базисе $\left\{h_{n}\right\}$ формулой

$$
\pi^{\mathscr{P}}(H(z))=e^{\gamma z} \prod_{i \geqslant 1} \frac{1+\beta_{i} z}{1-\alpha_{i} z} .
$$

Утверждение 2. (а) Пусть $P_{\mathscr{P}}(\lambda)$ - вероятность того, что заполнение диаграммы $\lambda$ независимыми случайными буквами с распределением $\mu_{1}$ оказывается $\mathscr{A}_{p}$-таблицей. Тогда

$$
P_{\mathscr{P}}(\lambda)=\pi^{\mathscr{P}}\left(s_{\lambda}\right)
$$

(b) Пусть $\lambda \in \mathbb{Y}_{n}$. Тогда

$$
\mu_{n}\left(w: \phi_{p}(w)=\lambda\right)=\operatorname{dim} \lambda \pi^{\mathscr{P}}\left(s_{\lambda}\right)=M_{n}^{\mathscr{P}}(\lambda) .
$$


Доказательство. См. [7, предложение 3 и теорема 1].

Существуют другие обобщения RSK-алгоритма (см. [10], [9]), для которых сохраняется справедливость утверждений 1 (a) и $2(\mathrm{~b})$, но не утверждения $1(\mathrm{~b})$.

2.2. Назовем множество $I \subset \mathscr{A}$ интервалом, если из неравенств

$$
a_{1}<a<a_{2}, \quad a_{1}, a_{2} \in I, a \in \mathscr{A},
$$

следует, что $a \in I$. Для удобства в дальнейшем будем считать упорядочения алфавита $\mathscr{A}$ такими, что $G$ образует интервал.

Обозначим символами $n_{i}(R), n_{j}^{\prime}(R)$ число букв $x_{i}$ и $y_{j}$ в $\mathscr{A}_{p}$-таблице $R$. Назовем типом $\mathscr{A}_{p}$-таблицы $R$ совокупность чисел

$$
\operatorname{type}(R):=\left(\left\{n_{i}(R)\right\},\left\{n_{j}^{\prime}(R)\right\}, m\right),
$$

где $m$ - число букв из $G$, стоящих в клетках таблицы $R$.

Напомним, что различным порядкам на $\mathscr{A}$ соответствуют различные отображения

$$
\phi_{p}: \mathscr{A}^{n} \rightarrow \mathbb{Y}_{n}
$$

Лемма 1. Пусть зафиксировань набор чисел $\left(\left\{n_{i}\right\} ;\left\{n_{j}^{\prime}\right\} ; m\right)$ и диаграмма $\lambda \in \mathbb{Y}_{n}$. Тогда величина

$$
\mu_{n}\left(w \in \mathscr{A}^{n}: \phi_{p}(w)=\lambda ; \operatorname{type}(R(w))=\left(\left\{n_{i}\right\} ;\left\{n_{j}^{\prime}\right\} ; m\right)\right)
$$

не зависит от упорядочения р.

Доказательство. Заметим, что вероятность совпадения двух букв из $G$ в слове $w$ равна 0 ; поэтому можно считать, что все буквы из $G$, входящие в слово $w$, различны. Пусть $g_{1}<\cdots<g_{m}-$ произвольные буквы из $G$. Рассмотрим набор из $|\lambda|$ букв $\Omega=\left(\left\{x_{i}\right\},\left\{y_{j}\right\}, g_{1}, \ldots, g_{m}\right)$, в который буква $x_{i}$ входит $n_{i}$ раз, а буква $y_{j}$ входит $n_{j}^{\prime}$ раз. Будем заполнять клетки диаграммы $\lambda$ буквами из $\Omega$ так, чтобы получалась $\mathscr{A}_{p}$-таблица. По $[9$, теорема 3$]$ число таких заполнений не зависит от упорядочения $p$. Обозначим это число символом $d\left(\left\{n_{i}\right\} ;\left\{n_{j}^{\prime}\right\} ; m\right)$. Каждому заполнению в силу утверждения 1 (а) соответствует ровно $\operatorname{dim} \lambda$ слов $w$, составленных из набора букв $\Omega$. Поэтому вероятность фиксированного заполнения диаграммы $\lambda$ равна

$$
\operatorname{dim} \lambda \prod_{i \geqslant 1} \alpha_{i}^{n_{i}} \prod_{j \geqslant 1} \beta_{j}^{n_{j}^{\prime}} \frac{1}{m !},
$$

где множитель $1 / m$ ! возникает из условия $g_{1}<\cdots<g_{m}$. Следовательно, искомая величина выражается формулой, не зависящей от упорядочения $p$ :

$$
\begin{aligned}
\mu_{n}\left(w \in \mathscr{A}^{n}: \phi_{p}(w)=\lambda ; \operatorname{type}(R(w))\right. & \left.=\left(\left\{n_{i}\right\} ;\left\{n_{j}^{\prime}\right\} ; m\right)\right) \\
& =\operatorname{dim} \lambda \frac{d\left(\left\{n_{i}\right\} ;\left\{n_{j}^{\prime}\right\} ; m\right)}{m !} \prod_{i \geqslant 1} \alpha_{i}^{n_{i}} \prod_{j \geqslant 1} \beta_{j}^{n_{j}^{\prime}} .
\end{aligned}
$$

Следствие. Распределение величин $\lambda_{i}^{\mathscr{P}}(n)-N_{x_{i}}(n), \lambda_{j}^{\prime P}(n)-N_{y_{j}}(n)$ не зависит от порядка $p$.

Зафиксируем на $\mathscr{A}$ порядок $p$, и пусть $I-$ интервал алфавита $\mathscr{A}$. Скажем, что алфавит $\mathscr{A}^{*}$ является укрупнением алфавита $\mathscr{A}$, если интервал $I$ заменяется одной новой буквой $z \in \mathscr{A}^{*}$ (остальные буквы не меняются). Будем считать, что $z \in L_{e}$ (вне зависимости от того, каким из множеств $L_{e}, L_{o}, G$ 
принадлежали буквы из $I)$, и сопоставим букве $z$ вероятность, равную $\mu_{1}(I)$. В этом случае отображение $\phi_{p}^{*}$ можно естественным образом определить как

$$
\phi_{p}^{*}: \mathscr{A}^{n} \rightarrow \mathbb{Y}_{n}
$$

т. е. на том же вероятностном пространстве, что и отображение $\phi_{p}$. В силу этого можно сравнивать длины строк случайных диаграмм Юнга, порождаемых алфавитами $\mathscr{A}$ и $\mathscr{A}^{*}$.

Лемма 2. Для любого $k>0$ выполняется неравенство

$$
\sum_{i=1}^{k} \lambda_{i}^{\mathscr{P}}(n) \leqslant \sum_{i=1}^{k} \lambda_{i}^{\mathscr{P} *}(n) .
$$

Доказательство. Вследствие утверждения 1(b)

$$
\sum_{i=1}^{k} \lambda_{i}^{\mathscr{P}}(n)=r_{k}(w), \quad \sum_{i=1}^{k} \lambda_{i}^{\mathscr{P}^{*}}(n)=r_{k}\left(w^{*}\right) .
$$

Заметим, что любая возрастающая (в смысле нашего определения) подпоследовательность слова $w \in \mathscr{A}^{n}$ переходит в возрастающую подпоследовательность соответствующего слова $w^{*} \in \mathscr{A}^{* n}$, так как новая буква $z$ принадлежит множеству $L_{e}$. Поэтому для любого $w$ выполнено неравенство

$$
r_{k}\left(w^{*}\right) \geqslant r_{k}(w) .
$$

Определим транспонирующее отображение, меняющее ролями строки и столбцы,

$$
\phi_{p^{t}}: \mathscr{A}^{n} \rightarrow \mathbb{Y}_{n}
$$

следующим образом. Рассмотрим на $\mathscr{A}$ упорядочение, обратное $p$ (будем обозначать его $\left.p^{t}\right)$, и будем считать, что $L_{e}^{t}=L_{o}, L_{o}^{t}=L_{e}$. Таким образом, параметры $\left\{\beta_{j}\right\}$ и $\left\{\alpha_{i}\right\}$ меняются местами; $\phi_{p^{t}}$ определяется обобщенным RSKалгоритмом, примененным к порядку $p^{t}$ и $L_{e}^{t}, L_{o}^{t}, G$. Будем обозначать через $\lambda^{t}$ диаграмму Юнга, транспонированную к $\lambda$.

Лемма 3. Для почти всех $w$

$$
\phi_{p}(w)=\phi_{p^{t}}(w)^{t}
$$

Доказательство. Если все буквы из $G$, входящие в $w$, различны (это условие вызвано формальной несимметричностью отношений $x_{1} \nearrow x_{2}$ и $\left.x_{1} \searrow x_{2}\right)$, то легко видеть, что возрастающая последовательность букв относительно $p$ и $L_{e} \cup L_{o}$ - это убывающая последовательность относительно $p^{t}, L_{e}^{t} \cup L_{o}^{t}$. Таким образом, лемма следует из утверждения 1(b).

2.3. Пусть $q_{1}, q_{2}, q_{3} \geqslant 0, q_{1}<q_{3}$ и $q_{1}+q_{2}+q_{3}=1$. Рассмотрим случайное блуждание частицы по множеству $\{0,1,2 \ldots\}$, в котором шаг вправо делается с вероятностью $q_{1}$, а шаг влево - с вероятностью $q_{3}$, за исключением точки 0 . Вначале частица находится в 0 . Обозначим символом $\Psi_{q_{3}, q_{1}}(n)$ положение частицы после $n$-го шага. Иначе говоря, $\Psi_{q_{3}, q_{1}}(n)-$ марковская цепь с переходной 
матрицей

$$
D=\left(\begin{array}{cccccc}
q_{3}+q_{2} & q_{1} & 0 & 0 & \ldots & \ldots \\
q_{3} & q_{2} & q_{1} & 0 & 0 & \ldots \\
0 & q_{3} & q_{2} & q_{1} & 0 & \ldots \\
\vdots & \ddots & \ddots & \ddots & \ddots & \ddots
\end{array}\right)
$$

и начальным вектором $\vec{a}_{0}=(1,0,0,0, \ldots)$.

Лемма 4. Существует константа $C$, не зависящая от $n$, такая, что

$$
\mathbf{E} \Psi_{q_{3}, q_{1}}(n)<C \text { для любого } n .
$$

Доказательство. Определим вектор $\vec{a}$ формулой

$$
\vec{a}=\left(2,2\left(q_{1} / q_{3}\right), 2\left(q_{1} / q_{3}\right)^{2}, \ldots\right) .
$$

Легко видеть, что $\vec{a} D=\vec{a}$. Кроме того, вектор $\vec{a}$ покомпонентно больше, чем начальный вектор этой марковской цепи $\vec{a}_{0}=(1,0,0,0, \ldots)$. Из неотрицательности элементов матрицы $D$ следует, что и $\vec{a} D^{n}$ будет покомпонентно больше, чем $\vec{a}_{0} D^{n}$, для любого $n$. Но $\vec{a} D^{n}=\vec{a}$, поэтому $\mathbf{E} \Psi_{q_{3}, q_{1}}(n)$ для любого $n$ будет ограничено числом $2 \sum_{i=0}^{\infty} i\left(q_{1} / q_{3}\right)^{i}$.

2.4. Зафиксируем порядок на $\mathscr{A}$. Пусть буквы $a, b \in L_{e}, a<b$, образуют интервал относительно этого порядка (т.е. $a$ и $b-$ соседние буквы) и $w \in \mathscr{A}^{n}$ - слово, подаваемое на вход RSK-алгоритма. Обозначим символом $w_{a, b}$ слово, полученное из $w$ вычеркиванием всех букв, кроме $a$ и $b$.

Назовем возможным преобразованием слова $w_{a, b}$ слово (обозначим его символом $\left.d_{w}\left(w_{a, b}\right)\right)$, которое образуют буквы $a$ и $b$, записанные в том порядке, в котором они выбиваются из первой строки в процессе действия обобщенного RSK-алгоритма на слове $w$; если какие-то буквы остались не выбитыми из первой строки, то допишем их в конец слова $d_{w}\left(w_{a, b}\right)$ в том порядке, в котором они стоят в первой строке.

Назовем суфбиксом слова $w=z_{1} \cdots z_{n}$ любое слово вида $z_{k} z_{k+1} z_{k+2} \cdots z_{n}$. Для всех суффиксов (включая пустой) слова $w_{a, b}$ определим разность числа букв $b$ и числа букв $a$, входящих в них. Максимальную из этих разностей назовем результатом и обозначим символом $\rho\left(w_{a, b}\right)$, а любой суффикс, на котором она достигается, будем называть максимальным.

Легко видеть, что если применять RSK-алгоритм непосредственно к слову $w_{a, b}$, то в первой строке останутся не выбитыми ровно $\rho\left(w_{a, b}\right)$ букв $b$.

Пример. Пусть $x_{1}<x_{2}<x_{3} \in L_{e}$ и $w=x_{2} x_{1} x_{3} x_{2} x_{1} x_{2} x_{3} x_{3} x_{2} x_{3} x_{1} x_{3} x_{2}$. Тогда $w_{x_{2} x_{3}}=x_{2} x_{3} x_{2} x_{2} x_{3} x_{3} x_{2} x_{3} x_{3} x_{2}$, максимальный суффикс слова $w_{x_{2}, x_{3}}$ состоит из 6 последних букв и $\rho\left(w_{x_{2}, x_{3}}\right)=2$. При этом

$$
d_{w}\left(w_{x_{2}, x_{3}}\right)=x_{2} x_{3} x_{2} x_{3} x_{2} x_{3} x_{2} x_{2} x_{3} x_{3} .
$$

Лемма 5. Для любого $w$

$$
\rho\left(d_{w}\left(w_{a, b}\right)\right) \leqslant \rho\left(w_{a, b}\right) .
$$

Доказательство. Шаг 1. Будем образовывать пары букв из слова $w_{a, b}$. В каждой паре будет одна буква $b$ и одна буква $a$, причем буква $a$ будет стоять в слове $w_{a, b}$ правее, чем буква $b$ из этой пары. Составим эти пары следующим образом: первой возьмем самую правую букву $a$ и поставим ей в соответствие 
ближайшую к ней слева букву $b$. Затем возьмем самую правую из еще не выбранных букв $a$ и поставим ей в пару ближайшую к ней слева букву $b$ из числа еще не выбранных. Проделаем эту процедуру максимально возможное число раз. Буквы $b$, не вошедшие в пары, будем называть черными, а вошедшие белыми.

Для слова $w_{x_{2}, x_{3}}$ из примера (перед леммой) разбиение на пары будет выглядеть следующим образом:

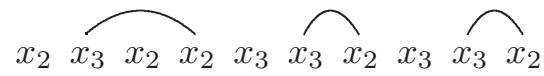

Шаг 2. Докажем, что ровно $\rho\left(w_{a, b}\right)$ букв $b$ не вошло в пары. Действительно, пусть число черных букв равно $\rho^{\prime}$. Рассмотрим суффикс, начинающийся с самой левой из черных букв. Каждая буква $a$ из этого суффикса должна быть сопоставлена с буквой $b$ из этого же суффикса. Поэтому разность числа букв $b$ и $a$ для него равна $\rho^{\prime}$. Значит, $\rho\left(w_{a, b}\right) \geqslant \rho^{\prime}$. С другой стороны, в максимальном суффиксе должно быть как минимум $\rho\left(w_{a, b}\right)$ черных букв. Поэтому $\rho^{\prime}=\rho\left(w_{a, b}\right)$.

Будем для удобства считать, что в ходе RSK-алгоритма выбивается сначала белая буква $b$ и, только если такой нет, то черная. Понятно, что это предположение никак не влияет на ход алгоритма.

Шаг 3. Покажем, что в каждой паре буква $b$ будет выбита раньше, чем соответствующая ей буква $a$. Будем доказывать это утверждение индукцией по числу пар. Рассмотрим самую левую пару. Буква $b$, входящая в нее, является первой белой буквой $b$ в слове, поэтому буква $a$ из этой пары обязана ее выбить. Для $k$-й слева пары рассуждение аналогично: в момент прихода буквы $a$ из этой пары белые буквы $b$ из предыдущих $k-1$ пар уже выбиты (по предположению индукции); поэтому пришедшая буква $a$ обязана выбить букву $b$ именно из своей пары (если она не была выбита раньше, что также возможно). Значит, порядок в паре будет тот же и после любого возможного преобразования слова $w_{a, b}$.

Следовательно, в результат слова $d_{w}\left(w_{a, b}\right)$ могут дать положительный вклад лишь черные буквы слова $w_{a, b}$, которых ровно $\rho\left(w_{a, b}\right)$. Поэтому результат слова не увеличивается после возможного преобразования.

\section{§3. Доказательства теорем}

3.1. Доказательство теоремы 2 для конечного $\mathscr{A}$. Докажем теорему 2 для частного случая, а именно, предположим, что среди $\alpha$ - и $\beta$-параметров имеется лишь конечное число ненулевых и что $\gamma=0$. В этом случае теорему достаточно доказать для случая, когда $K$ равно числу всех ненулевых $\alpha$-параметров, а $L$ - числу всех ненулевых $\beta$-параметров. Таким образом, $\mathscr{A}=$ $\left\{x_{1}, \ldots, x_{K}\right\} \cup\left\{y_{1}, \ldots, y_{L}\right\}$.

В силу следствия из леммы 1 утверждения теоремы достаточно доказать для какого-то одного порядка. Упорядочим алфавит $\mathscr{A}$ следующим образом:

$$
x_{1}<\cdots<x_{K}<y_{L}<y_{L-1}<\cdots<y_{1} .
$$

Будем применять к случайному слову $w \in \mathscr{A}^{n}$ обобщенный RSK-алгоритм. Обозначим символом $\xi_{j}^{i}(n)$ число букв $x_{j}$ в $i$-й строке получающейся $\mathscr{A}$-таблицы. Легко видеть, что при введенном порядке $\xi_{j}^{i}(n)=0$, если $i>j$. 
Последовательность случайных величин $\{\psi(n)\}$ назовем L-ограниченной, ecли существует константа $C$, не зависящая от $n$ и такая, что

$$
\mathbf{E}|\psi(n)|<C \quad \text { для любого } n \text {. }
$$

Будем обозначать любые последовательности L-ограниченных случайных величин символом $\{L(n)\}$. Заметим, что выполнены соотношения

$$
\{L(n)\}+\{L(n)\}=\{L(n)\}, \quad\{L(n)\}-\{L(n)\}=\{L(n)\} .
$$

Сначала докажем утверждение теоремы для строк. Будем вести индукцию по строкам.

1) Первая строка. Оценка снизу. Заметим, что все буквы $x_{1}$ стоят в первой строке. Поэтому

$$
\lambda_{1}^{\mathscr{P}}(n) \geqslant N_{x_{1}}(n) .
$$

Оценка сверху. Посмотрим, по каким правилам меняется число $\xi_{k}^{1}(n)$ при $k \geqslant 2$. Увеличиваться оно может только в случае появления буквы $x_{k}$, что происходит с вероятностью $\alpha_{k}$. Если же появляется $x_{k-1}$, что происходит с вероятностью $\alpha_{k-1}>\alpha_{k}$, и $\xi_{k}^{1} \neq 0$, то $\xi_{k}^{1}$ обязано уменьшиться на 1 . В силу леммы 4 это означает, что последовательность $\xi_{k}^{1}(n)$ L-ограничена. Из определения $\mathscr{A}$-таблицы следует, что в каждой строке не больше одной буквы $y_{j}$. Поэтому

$$
\lambda_{1}^{\mathscr{P}}(n)=N_{x_{1}}(n)+\{L(n)\} .
$$

2 ) Зафиксируем $l \leqslant K$. Пусть утверждение теоремы верно для первых $l-1$ строк. Докажем его для $l$-й.

Оценка снизу. Буква $x_{l}$ не может попасть ниже $l$-й строки. С другой стороны, по уже доказанному выше $l$-й строки может быть лишь L-ограниченное количество букв $x_{l}$. Поэтому

$$
\lambda_{l}^{\mathscr{P}}(n) \geqslant N_{x_{l}}(n)-\{L(n)\} .
$$

Оценка сверху. Обозначим символом $w^{0}$ слово на входе обобщенного RSK-алгоритма и символами $w^{1}, w^{2}, \ldots$ - слова, в которые записываются буквы в том порядке, в котором они выбиваются из первой, второй, ... строк.

Докажем L-ограниченность величины $\xi_{k}^{l}(n)$ при $k>l$. Заметим, что $w_{x_{k-1}, x_{k}}^{i-1}$ - это последовательность букв $x_{k-1}$ и $x_{k}$, поступающих в $i$-ю строку, а $w_{x_{k-1}, x_{k}}^{i}$ - последовательность букв $x_{k-1}$ и $x_{k}$, выбиваемых из $i$-й строки. Поэтому слово $w_{x_{k-1}, x_{k}}^{i}$ является возможным преобразованием слова $w_{x_{k-1}, x_{k}}^{i-1}$, из которого вычеркнуты те буквы $x_{k-1}$ и $x_{k}$, которые остались не выбитыми из $i$-й строки. Но таких букв по предположению индукции L-ограниченное число. Поэтому из леммы 5 следует, что

$$
\rho\left(w_{x_{k-1}, x_{k}}^{i}\right) \leqslant \rho\left(w_{x_{k-1}, x_{k}}^{i-1}\right)+\{L(n)\}, \quad i=1, \ldots, l-1 .
$$

Суммируя неравенства (3.1) по $i=1, \ldots, l-1$, получаем $\rho\left(w_{x_{k-1}, x_{k}}^{l-1}\right) \leqslant \rho\left(w_{x_{k-1}, x_{k}}^{0}\right)$ $+\{L(n)\}$. Кроме того, $\rho\left(w_{x_{k-1}, x_{k}}^{0}\right)=\{L(n)\}$ в силу леммы 4. Рассуждая так же, как при доказательстве оценки сверху для первой строки, приходим к неравенству $\xi_{k}^{l}(n) \leqslant \rho\left(w_{x_{k-1}, x_{k}}^{l-1}\right)$. Следовательно, $\xi_{k}^{l}(n)$ есть L-ограниченная величина для любого $k>l$. Как уже было замечено, ни в одной строке не может быть больше одной буквы $y_{j}$ (для каждого $j$ ). Значит, $\lambda_{l}^{\mathscr{P}}(n)=N_{x_{l}}(n)+\{L(n)\}$. 
Для оценки величин $\lambda_{1}^{\prime \mathscr{P}}(n), \ldots, \lambda_{L}^{\prime P}(n)$ рассмотрим транспонирующее отображение. $\mathrm{K}$ мере, задаваемой параметрами $\mathscr{P}^{t}=\left(\left\{\beta_{j}\right\},\left\{\alpha_{i}\right\}, \gamma\right)$, мы можем применить уже доказанные оценки для строк. В силу леммы 3

$\lambda_{1}^{\prime \mathscr{P}}(n)=\lambda_{1}^{\mathscr{P}^{t}}(n)=N_{y_{1}}(n)+\{L(n)\}, \quad \ldots, \quad \lambda_{L}^{\prime \mathscr{P}}(n)=\lambda_{L}^{\mathscr{P}^{t}}(n)=N_{y_{L}}(n)+\{L(n)\}$.

3.2. Доказательство теоремы 2 для общего случая. Пусть $\mathscr{P}=\left(\left\{\alpha_{i}\right\}\right.$, $\left.\left\{\beta_{j}\right\}, \gamma\right)$ - набор параметров, удовлетворяющих условию строгой монотонности (1.1). Докажем сначала теорему для длин строк - утверждение для столбцов получится отсюда с помощью транспонирующего отображения.

В силу следствия из леммы 1 оценки сверху и снизу на величину $\mathbf{E}\left(\lambda_{i}^{\mathscr{P}}(n)-\right.$ $\left.N_{x_{i}}(n)\right)$ можно доказывать для различных упорядочений алфавита $\mathscr{A}$. Введем порядок $p_{1}$ на $\mathscr{A}$ :

$$
x_{1}<x_{2}<\cdots<y_{1}<y_{2}<\cdots<G .
$$

Оиенка снизу. Для порядка $p_{1}$ буквы $x_{1}, \ldots, x_{K}$ эволюционируют вне зависимости от других букв, поэтому оценка

$$
\lambda_{i}^{\mathscr{P}}(n) \geqslant N_{x_{i}}(n)+\{L(n)\}, \quad i=1, \ldots, K,
$$

доказывается аналогично случаю конечного $\mathscr{A}$.

Идея доказательства оценки сверху состоит в сведении общего случая к случаю конечного числа параметров с помощью операции укрупнения. Порядок $p_{2}$, используемый для оценки сверху, определим так, чтобы были возможны операции укрупнения, предписанные приведенными ниже шагами (т. е. буквы, которые будет нужно отождествить, должны образовывать интервалы относительно порядка $\left.p_{2}\right)$. Нашей целью является получение после нескольких укрупнений конечного числа параметров, причем $K$ первых по величине $\alpha$-параметров не должны измениться и все $\alpha$-параметры должны быть различны.

1) Пусть число $\alpha$-параметров в $\mathscr{P}$ бесконечно. Рассмотрим два случая:

(a) Допустим, что существует $l \in \mathbb{N}$, такое, что выполнено неравенство

$$
\sum_{i=l+1}^{\infty} \alpha_{i}<\alpha_{K}
$$

и для любого $r \leqslant l$

$$
\sum_{i=l+1}^{\infty} \alpha_{i} \neq \alpha_{r}
$$

В этом случае отождествим буквы $x_{l+1}, x_{l+2}, x_{l+3}, \ldots$

(b) Если такого $l$, как требуется в п. (а), не существует, то, как легко видеть, найдутся $l_{1}, m_{1} \in \mathbb{N}$, такие, что для некоторого $r \leqslant l_{1}$

$$
\sum_{i=l_{1}+1}^{\infty} \alpha_{i}=\alpha_{r}<\alpha_{K}
$$

и выполняются условия

$$
\alpha_{r}>\sum_{i=l_{1}+1}^{l_{1}+m_{1}} \alpha_{i}>\alpha_{r+1}, \quad \sum_{i=l_{1}+m_{1}+1}^{\infty} \alpha_{i}<\alpha_{l_{1}} .
$$

В этом случае отождествим буквы $x_{l_{1}+1}, \ldots, x_{l_{1}+m_{1}}$, а также (отдельно) $x_{l_{1}+m_{1}+1}, x_{l_{1}+m_{1}+2}, x_{l_{1}+m_{1}+3}, \ldots$.

После проведенных операций укрупнения остается конечное число попарно различных $\alpha$-параметров. Обозначим минимальный из них символом $\alpha_{R}$. 
$2)$ Если число $\beta$-параметров бесконечно, то выберем $l_{2}$ так, чтобы было выполнено условие

$$
\sum_{i=l_{2}+1}^{\infty} \beta_{i}<\alpha_{R}
$$

и отождествим буквы $y_{l_{2}+1}, y_{l_{2}+2}, y_{l_{2}+3}, \ldots$ Напомним, что после такой операции укрупнения возникает новый $\alpha$-параметр, который в силу (3.2) меньше других $\alpha$-параметров. Обозначим его символом $\alpha_{R+1}$.

3) Если $\gamma>0$, то легко видеть, что найдутся $m \in \mathbb{N}$ и $\delta_{1}>\cdots>\delta_{m} \in \mathbb{R}$, такие, что

$$
\delta_{1}+\cdots+\delta_{m}=0, \quad \gamma / m+\delta_{1}<\alpha_{R+1}, \quad \gamma / m+\delta_{m}>0 .
$$

Разобьем $G$ на непересекающиеся интервалы длины $\gamma / m+\delta_{1}, \gamma / m+\delta_{2}, \ldots, \gamma / m+$ $\delta_{m}$ и отождествим точки в каждом из этих интервалов. В результате такой операции укрупнения возникнет $m$ новых $\alpha$-параметров, а из выбора $\delta_{i}$ следует, что эти параметры будут попарно различны и меньше всех полученных на предыдущих шагах.

Таким образом, каждый набор параметров $\mathscr{P}$ можно операциями укрупнения, описанными в пп. 1)-3), свести к случаю конечного числа параметров, причем $K$ первых по величине $\alpha$-параметров при этом не меняются. Обозначим полученный набор параметров символом $\mathscr{P}^{*}$. Напомним, что величины $\lambda_{i}^{\mathscr{P}^{*}}(n)$ естественным образом определены на $\left(\mathscr{A}^{n}, \mu_{n}\right)$.

Оченка сверху.

1) Первая строка. По лемме 2 имеем $\lambda_{1}^{\mathscr{P}}(n) \leqslant \lambda_{1}^{\mathscr{P}}(n)$. Так как $\mathscr{P}^{*}$ соответствует случаю конечного алфавита, то по уже доказанному $\lambda_{1}^{\mathscr{P} *}(n) \leqslant$ $N_{x_{1}}(n)+\{L(n)\}$. Следовательно, $\lambda_{1}^{\mathscr{P}}(n) \leqslant N_{x_{1}}(n)+\{L(n)\}$.

$2)$ Докажем утверждение для $l$-й строки. По лемме 2

$$
\lambda_{1}^{\mathscr{P}}(n)+\cdots+\lambda_{l}^{\mathscr{P}}(n) \leqslant \lambda_{1}^{\mathscr{P}^{*}}(n)+\cdots+\lambda_{l}^{\mathscr{P}^{*}}(n) .
$$

Из уже доказанного в разд. 3.1 имеем

$$
\lambda_{1}^{\mathscr{P}^{*}}(n)+\cdots+\lambda_{l}^{\mathscr{P}^{*}}(n) \leqslant N_{x_{1}}(n)+\cdots+N_{x_{l}}(n)+\{L(n)\} .
$$

Из оценки снизу получаем, что

$$
\lambda_{1}^{\mathscr{P}}(n)+\cdots+\lambda_{l-1}^{\mathscr{P}}(n) \geqslant N_{x_{1}}(n)+\cdots+N_{x_{l-1}}(n)+\{L(n)\} .
$$

Следовательно,

$$
\lambda_{l}^{\mathscr{P}}(n) \leqslant N_{x_{l}}(n)+\{L(n)\} .
$$

Доказательство утверждения теоремы для длин столбцов вытекает из утверждения для строк аналогично случаю конечного алфавита, что завершает доказательство теоремы 2.

3.3. Доказательство теоремы 1. Простым вычислением характеристических функций можно показать, что

$$
\begin{aligned}
\eta_{n}:=\left(\frac{N_{x_{1}}(n)-\alpha_{1} n}{\sqrt{n}}, \ldots, \frac{N_{x_{K}}(n)-\alpha_{K} n}{\sqrt{n}},\right. & \left.\frac{N_{y_{1}}(n)-\beta_{1} n}{\sqrt{n}}, \ldots, \frac{N_{y_{L}}(n)-\beta_{L} n}{\sqrt{n}}\right) \underset{\text { law }}{\longrightarrow} \eta,
\end{aligned}
$$


где символом $\eta$ обозначена многомерная гауссова случайная величина с нулевым средним и матрицей ковариаций $C$, задаваемой формулой

$$
C=\left(\begin{array}{cccccccc}
\alpha_{1}-\alpha_{1}^{2} & -\alpha_{1} \alpha_{2} & -\alpha_{1} \alpha_{3} & \ldots & -\alpha_{1} \alpha_{K} & -\alpha_{1} \beta_{1} & \ldots & -\alpha_{1} \beta_{L} \\
-\alpha_{2} \alpha_{1} & \alpha_{2}-\alpha_{2}^{2} & -\alpha_{2} \alpha_{3} & \ldots & -\alpha_{2} \alpha_{K} & -\alpha_{2} \beta_{1} & \ldots & -\alpha_{2} \beta_{L} \\
\vdots & \vdots & \ddots & \vdots & \vdots & \vdots & \vdots & \vdots \\
-\alpha_{K} \alpha_{1} & -\alpha_{K} \alpha_{2} & \ldots & \ldots & \alpha_{K}-\alpha_{K}^{2} & -\alpha_{K} \beta_{1} & \ldots & -\alpha_{K} \beta_{L} \\
-\beta_{1} \alpha_{1} & -\beta_{1} \alpha_{2} & \ldots & \ldots & -\beta_{1} \alpha_{K} & \beta_{1}-\beta_{1}^{2} & \ldots & -\beta_{1} \beta_{L} \\
\vdots & \vdots & \vdots & \vdots & \vdots & \vdots & \ddots & \vdots \\
-\beta_{L} \alpha_{1} & -\beta_{L} \alpha_{2} & \ldots & \ldots & -\beta_{L} \alpha_{K} & -\beta_{1} \beta_{L} & \ldots & \beta_{L}-\beta_{L}^{2}
\end{array}\right) .
$$

Легко видеть, что

$$
\psi_{n}:=\frac{(\{L(n)\}, \ldots,\{L(n)\})}{\sqrt{n}} \underset{\text { prob }}{\longrightarrow} 0
$$

для произвольных L-ограниченных последовательностей случайных величин (обозначенных через $\{L(n)\})$. Хорошо известно (см., например, [13, теорема $3.1])$, что $\eta_{n}^{0} \underset{\text { law }}{\longrightarrow} \eta^{0}$ и $\psi_{n}^{0} \underset{\text { prob }}{\longrightarrow} 0$ влечет за собой

$$
\eta_{n}^{0}+\psi_{n}^{0} \underset{\text { law }}{\longrightarrow} \eta^{0}
$$

Поэтому

$$
\begin{aligned}
&\left(\frac{\lambda_{1}^{\mathscr{P}}(n)-\alpha_{1} n}{\sqrt{n}}, \ldots, \frac{\lambda_{K}^{\mathscr{P}}(n)-\alpha_{K} n}{\sqrt{n}},\right. \\
&\left.\frac{\lambda_{1}^{\prime \mathscr{P}}(n)-\beta_{1} n}{\sqrt{n}}, \ldots, \frac{\lambda_{L}^{\prime \mathscr{P}}(n)-\beta_{L} n}{\sqrt{n}}\right)=\eta_{n}+\psi_{n} \underset{\text { law }}{\longrightarrow} \eta .
\end{aligned}
$$

Таким образом, теорема 1 доказана.

\section{ЛитерАТУРА}

[1] E. Thoma, Die unzerlegbaren, positive-definiten Klassenfunktionen der äbzahlbar unendlichen symmetrischen Gruppe, Math. Z., 85 (1964), 40-61.

[2] V. Feray, P.-L. Méliot, Asymptotics of q-Plancherel measures, http://arxiv.org/abs/ 1001.2180 .

[3] P.-L. Méliot, A central limit theorem for the characters of the infinite symmetric group and of the infinite Hecke algebra, http://arxiv.org/abs/1105.0091.

[4] А. М. Вершик, С. В. Керов, Асимптотическая теория характеров симметрической групnьь, Функц. анал. и его прил., 15:2 (1982), 15-27.

[5] S. Kerov, A. Okounkov, G. Olshanski, The boundary of the Young graph with Jack edge multiplicities, Internat. Math. Res. Notices, 1998:4, 173-199.

[6] S. Kerov, G. Olshanski, A. Vershik, Harmonic analysis on the infinite symmetric group, Invent. Math., 158:4 (2004), 551-642.

[7] S. V. Kerov, A. M. Vershik, The characters of the infinite symmetric group and probability properties of the Robinson-Schensted-Knuth algorithm, SIAM J. Algebraic Discrete Methods, 7:1 (1986), 116-124.

[8] И. Макдональд, Симметрические функиии и многочлены Холла, Мир, М., 1984.

[9] A. Regev, T. Seeman, Shuffle-invariance of the super-RSK algorithm, Adv. Appl. Math., 28:1 (2002), 59-81. 
[10] A. Berele, A. Regev, Hook Young diagrams with applications to combinatorics and representations of Lie superalgebras, Adv. in Math., 64:2 (1987), 118-175.

[11] W. Fulton, Young Tableaux, Cambridge Univ. Press, Cambridge, 1997.

[12] C. Schensted, Longest increasing and decreasing subsequences, Canad. J. Math., 13 (1961), 179-191.

[13] P. Billingsley, Convergence of Probability Measures, Wiley, New York, 1999.

[14] S. V. Kerov, Asymptotic Representation Theory of the Symmetric Group and its Applications in Analysis, Translations of Math. Monographs, vol. 219, Amer. Math. Soc., Providence, RI, 2003.

[15] K. Johansson, Discrete orthogonal polynomial ensembles and the Plancherel measure, Ann. of Math., 153:1 (2001), 259-296.

Московский государственный университет,

Поступило в редакцию механико-математический факультет 20 апреля 2011 г.

e-mail: a_bufetov@mail.ru 